\title{
Skin Metastasis of Gastrointestinal Stromal Tumors: A Case Series and Literature Review
}

This article was published in the following Dove Press journal:

Cancer Management and Research

\section{Peng Liu* \\ Fengbo Tan* \\ Heli Liu (D) \\ Jie $\mathrm{Ge}$ \\ Sheng Liu \\ Tianxiang Lei $\mathbb{D}$ \\ Xianhui Zhao}

Department of Gastrointestinal Surgery, Xiangya Hospital, Central South University, Changsha 410008, Hunan, People's Republic of China

*These authors contributed equally to this work
Correspondence: Heli Liu Department of Gastrointestinal Surgery,

Xiangya Hospital, Central South

University, Changsha 410008, Hunan,

People's Republic of China

Tel $+86 \quad 13874967285$

Email heliliu@csu.edu.cn
Background: Gastrointestinal stromal tumors (GISTs) extremely and rarely metastasize to the skin, and such metastases have not been well characterized.

Methods: Retrospective analysis of clinicopathological data of patients with skin metastasis of a GIST (SM-GIST) admitted to Xiangya Hospital (Changsha, Hunan, China) and literature review were conducted.

Results: Including our 4 cases, a total of 17 cases have been reported to date. The mean age of the patients was 55.4 years (29 70 years) and there was not sex predominance (male 10 and female 7). Primary tumors were often located in the stomach $(n=9)$, duodenum $(n=2)$ and small bowel $(n=2)$. Meanwhile, SM-GIST mainly occurred in head and face $(n=6)$, extremities $(n=6)$, followed by abdomen wall $(n=5)$, back $(n=3)$ and chest $(n=2)$. Mutation analysis revealed that the frequency of wild-type GIST (WT-GIST), exon 9, 11 and 13 mutations was 6, 1, 4 and 1, respectively. The average time to SM-GIST was 4.22 years, specifically 4.59 years in gastric and 3.8 years in non-gastric. Moreover, for the resection only group (including chemotherapy), such average time was 3.63 years, while for the combined group (resection and tyrosine kinase inhibitors (TKIs)), it was about 4.74 years. The mean survival was approximately 6.2 years. However, after the diagnosis of SM-GIST, survival was only about 1.69 years.

Conclusion: SM-GIST is a rare malignant condition. Non-gastric GIST, surgery without TKIs, high invasiveness and tumor burden, and molecular subtype (mutation in exon 9, 11 and wild-type) may be conducive to the development of SM-GIST. Additionally, it is also a sign of poor prognosis.

Keywords: gastrointestinal stromal tumor, skin metastasis, tyrosine kinase inhibitors

\section{Introduction}

Gastrointestinal stromal tumors (GISTs) are the most common mesenchymal neoplasms of the GI tract with an estimated annual incidence of around 10 per million worldwide. ${ }^{1,2}$ The primary tumors are predominantly located in stomach $(60 \%)$ and small intestine (25\%), but also can arise in other organs, such as rectum and esophagus. ${ }^{2}$ The morphology usually includes spindle cell type (70\%), followed by epithelioid (20\%) and mixed cell type $(10 \%),{ }^{3}$ and are characterized by staining for CD117 and DOG1 (95\% and 98\%, respectively). ${ }^{4,5}$ The carcinogenesis of GIST is mainly associated with activating mutations in KIT protooncogene, receptor tyrosine kinase $(K I T)$ or platelet-derived growth factor receptor alpha (PDGFRA), and also can harbor mutations in $B R A F, N F 1$ and $S D H$ complex called "wild-type" GISTs (WT-GISTs). ${ }^{6,7}$

At the time of diagnosis, up to $20 \%$ of patients have developed metastases which results in poor prognosis. ${ }^{8}$ The sites most commonly affected by GIST metastasis are 
abdominal cavity and liver, but it can also infrequently metastasize extra-abdominally to bone, pleura and soft tissue., ${ }^{2,9}$ Although several reports of skin (including cutaneous and subcutaneous tissue) metastasis of a GIST (SM-GIST) have been published in the literature, it has not been well characterized to date. ${ }^{10-18}$ Due to its challenging diagnosis, it is extremely necessary to summarize and analyze such cases in order to provide clinical guidance for the management of patients.

\section{Materials and Methods}

As of March 1, 2020, a total of 4 cases with SM-GIST had been identified from the GIST database of Xiangya Hospital of Central South University (Changsha, Hunan, China) which contains 881 GIST patients, and retrospective review of these 4 cases (Cases 1 to 4 ) was performed. The expression of CD117, CD34 and DOG1 was detected using a standard immunohistochemical method for all cases, and molecular analysis included KIT exon 9, 11, 13, 17 and PDGFRA exon 12, 18 was performed for three cases. We also reviewed 13 cases reported in the literature involving this uncommon metastatic pattern.

\section{Results \\ Case Series Case I}

A 70-year-old male underwent tumor wedge gastric resection and was diagnosed as gastric stromal tumor (highrisk) 5 years ago. After surgery, the patient was treated with imatinib, but the therapy was discontinued due to severe hematotoxicity. At the current admission, imaging examination revealed tumor recurrence and metastasis to the stomach, liver and abdominal cavity, as well as the presence of multiple subcutaneous nodules in the left anterior abdominal wall. Due to suspected subcutaneous metastasis, excision biopsy was performed, and pathological examination revealed a tumor with spindled morphology, which stained positive for CD117, DOG1 and CD34 (Figure 1). Additional molecular analysis confirmed mutations in KIT exon 11 and 13 (Figure 2). The diagnosis was established by consistent histopathological features and mutations with GIST. Currently, the patient continues to be treated intermittently with imatinib due to hematotoxicity and refusal of sunitinib, and is experiencing stable disease.

\section{Case 2}

A 52-year-old male presented with an isolated and painless subcutaneous nodule $(3 * 3 \mathrm{~cm})$ in the right upper abdominal wall. His medical history revealed a high-risk duodenum GIST (size $22 * 20 * 5 \mathrm{~cm}$, mitotic index $>5 / 10 \mathrm{HPF}$ ) and resection of tumor was performed 5 years earlier. However, adjuvant therapy with imatinib was not given after surgery duo to economic factors. One year later, imaging examination revealed that the tumor had metastasized into peritoneal cavity and lymph nodes. Resection of an abdominal mass was then performed and pathological examination revealed a tumor with classic spindled morphology, which stained for CD117, CD34 and DOG1. After that, the patient switched to sunitinib due to imatinib-resistance and at present the disease is in a stable state.

\section{Case 3}

A 29-year-old female underwent surgery for gastric GIST 10 years earlier in another hospital and was not treated with imatinib after surgery. Unfortunately, the details and specimen of the primary tumor were unavailable. Six months prior to the hospital visit, the patient experienced recurrence and multiple metastases in liver and the abdominal cavity. In addition, a subcutaneous nodule was found in her back and nodulectomy was performed. The subsequent histopathological analysis revealed a tumor with epithelioid morphology, which stained positive for DOG1, CD117 and CD34 but negative for succinate dehydrogenase B (SDHB) (Figure 3). Molecular analysis showed that there was no mutation in KIT and PDGFRA. According to the above results, the patient was diagnosed with SDH-deficient "wild-type" GIST. At present, treatment with imatinib has been stopped and switched to sunitinib due to progressive disease.

\section{Case 4}

A 51-year-old female presented to the department of gastrointestinal surgery with abdominal pain and mass 5 years earlier. The imagining examination revealed a retroperitoneal mass which was difficult to biopsy by minimally invasive surgery. Then, the resection of the retroperitoneal mass was performed and the tumor mass was diagnosed as GIST $(7 * 5 * 2.8 \mathrm{~cm})$ composed of spindle cells. The mitotic index was up to 10/50 HPF (high power field) and the immunohistochemistry (IHC) analysis showed that the tumor was positive for CD34 and DOG1. The patients had undergone treatment postoperatively with imatinib for 3 years and stopped medicinal treatment due to being disease-free. 

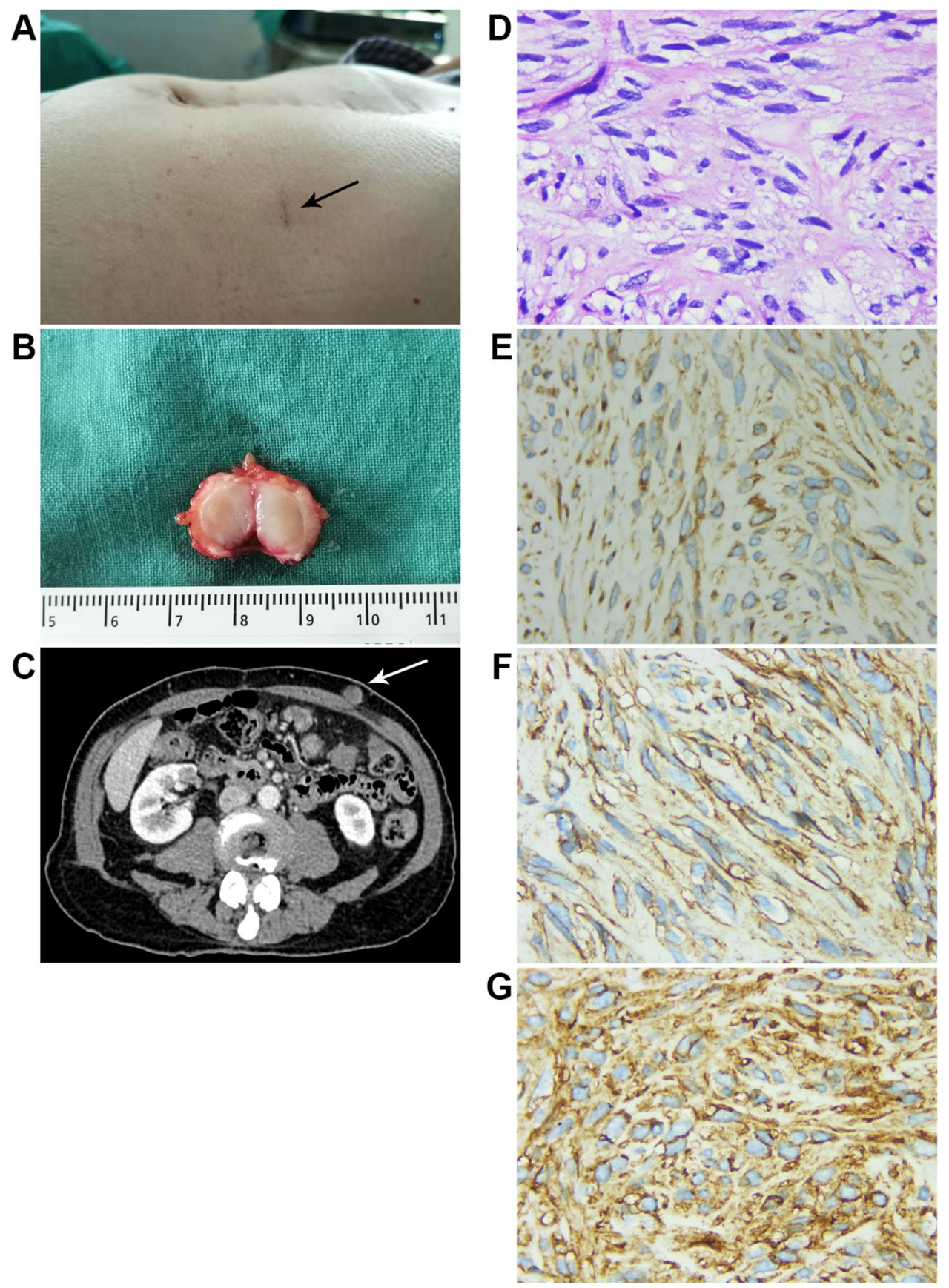

Figure I (A) Subcutaneous nodules in the left anterior abdominal wall (arrow); (B) Surgical specimens after resection of subcutaneous nodule; (C) Abdominal computed tomography showed a subcutaneous nodule in the left anterior wall (arrow); (D) Histology of the subcutaneous nodule showed that the tumor cells were spindled (H\&E staining, ×400); (E-G) CDII7, DOGI and CD34 immunohistochemistry is positive in the subcutaneous metastasis, respectively.

After 18 months of withdrawal of the medication, the patient went to general surgery due to the development of enlarging subcutaneous nodules on the back and right thigh. A computed tomography (CT) scan also revealed retroperitoneal recurrence and pulmonary metastasis. An excisional biopsy of the back nodule was performed as skin metastases were suspected. The histological features were compatible with the known primary tumor, but the tumor only stained positive for CD34 and molecular analysis showed no mutation in KIT/PDGFRA (Figure 4). In view of the confirmed primary tumor and the efficacy of imatinib, the GIST subcutaneous metastasis was established, and the patient was treated with imatinib. During the 6-month follow-up, the pulmonary lesion shrank, but there was no change in the subcutaneous lesion. 

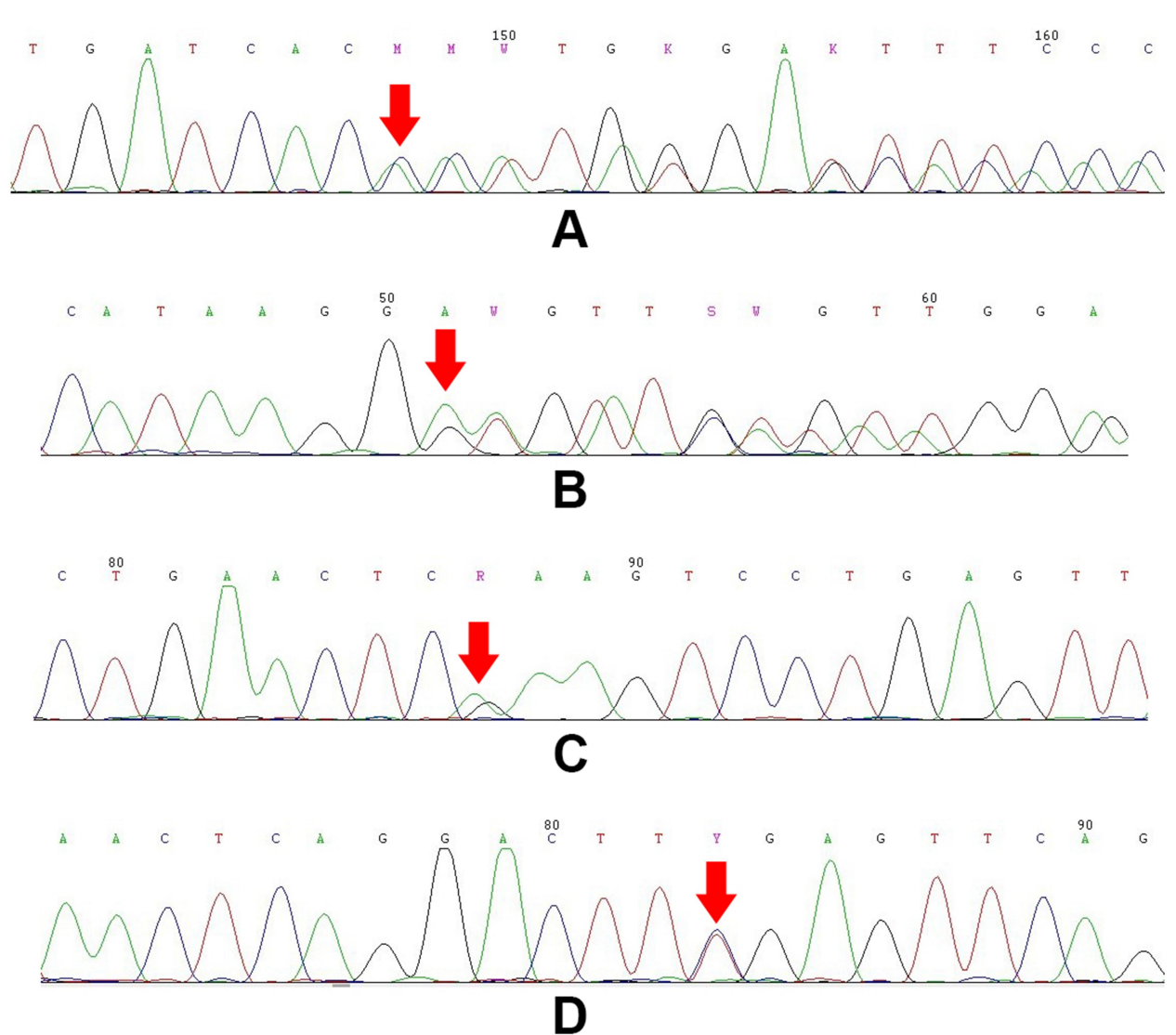

Figure 2 Sequencing results of KIT exon II and I3. The obvious double peaks in the sequencing diagram demonstrates there is mutation (red arrow). (A) and (B) Forward and reverse sequencing of KIT exon II (c.1740_I74I ins(p.P577-H580dup)); (C) and (D) Forward and reverse sequencing of KIT exon I3 (c.1924A>G(p.K642E)).
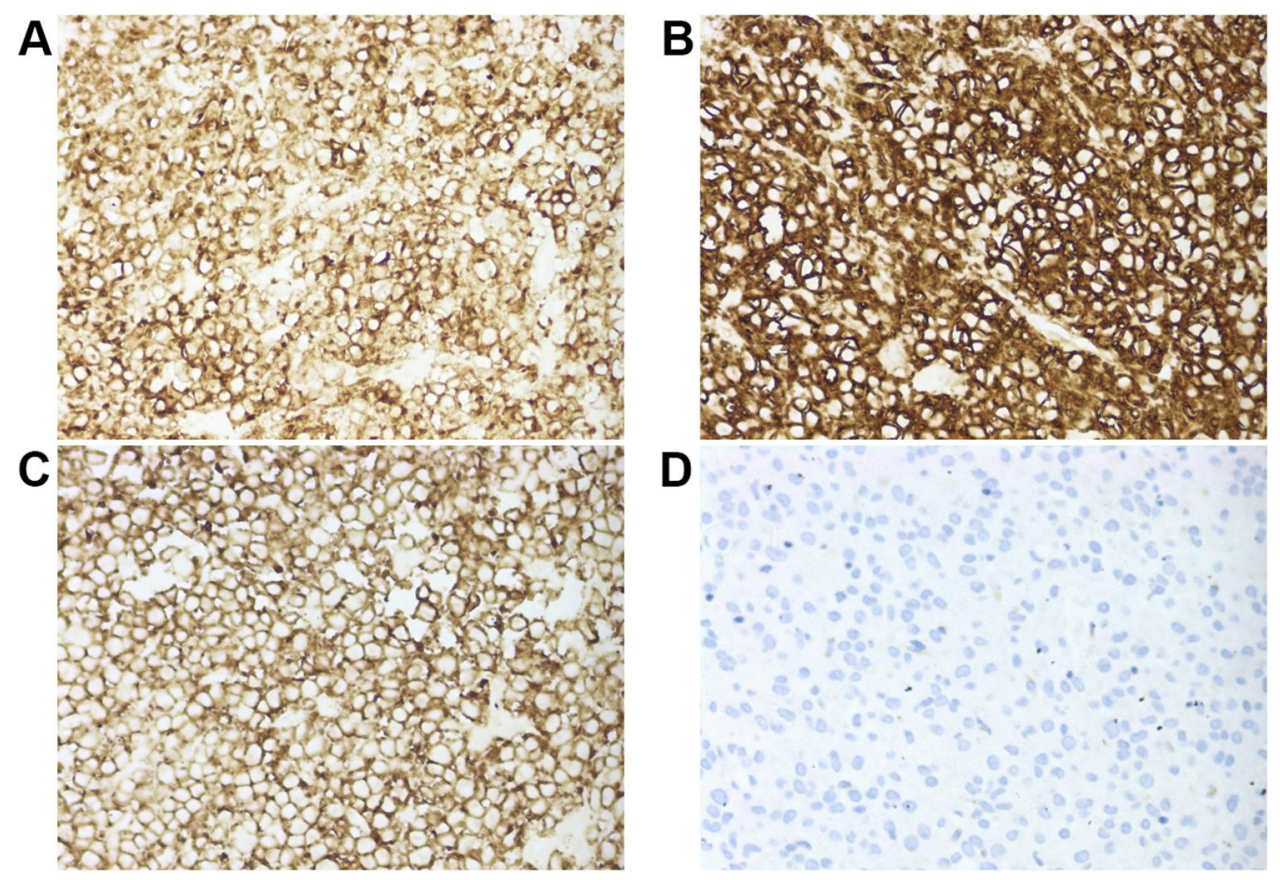

Figure 3 Immunohistochemical staining of SM-GIST (Case 3) showed the following: (A) CDII7 (positive, $\times 100$ ); (B) CD34 (positive, $\times 100)$; (C) DOGI (positive, $\times 100$ ); (D) SDHB (negative, $\times 100$ ). 

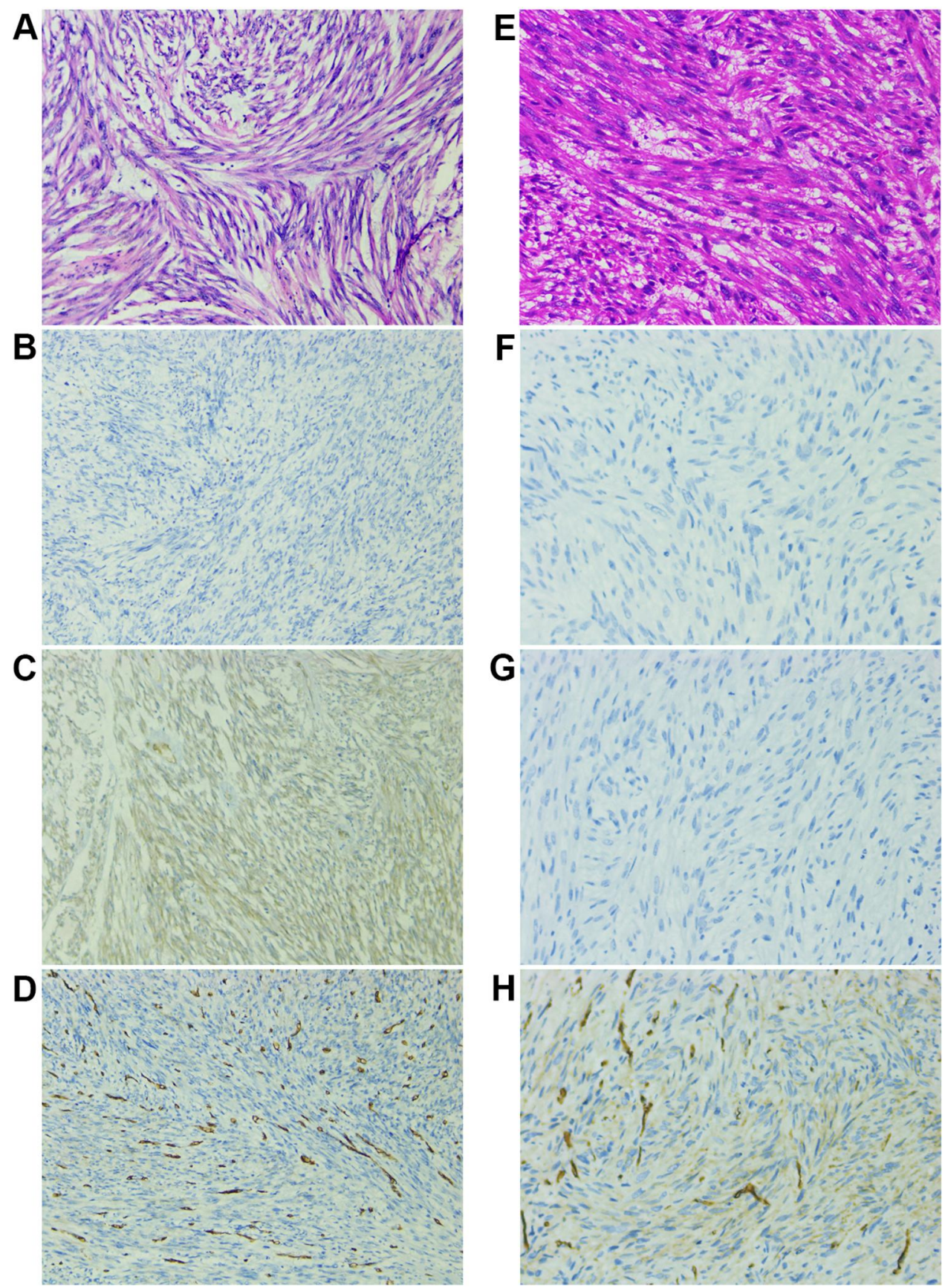

Primary tumor

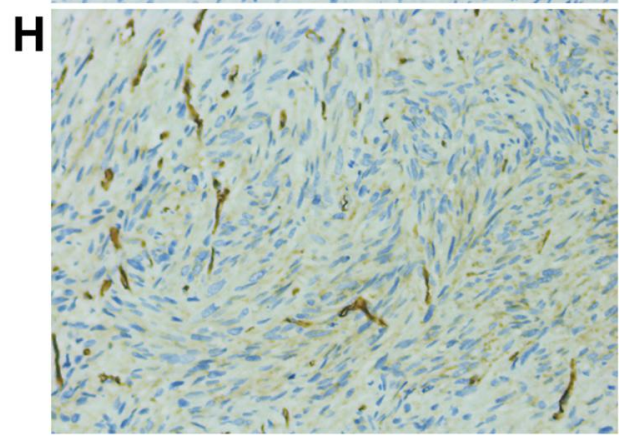

\section{SM-GIST}

Figure 4 Immunohistochemical staining of a primary tumor and skin metastasis. Primary tumor: (A) H\&E staining, $\times 100$; Immunohistochemical staining showed the following: (B) CDII7 (negative, × 100); (C) DOGI (positive, ×100); (D) CD34 (positive, $\times 100$ ). Skin metastasis: (E) H\&E staining, $\times 100$; Immunohistochemical staining showed the following: (F) CDII7 (negative, $\times 200$ ); (G) DOGI (negative, $\times 200$ ); (H) CD34 (positive, $\times 200$ ).

\section{Literature Review}

A review of the literature identified 9 articles describing 13 SM-GIST cases. ${ }^{10-18}$ Therefore, combined with the 4 cases presented here, a total of 17 cases of SM-GIST have now been reported (Table 1).
In these 17 cases, the average age at the diagnosis of SM-GIST was 55.4 years (29 70 years) with a similar male-female ratio (10 vs 7). Primary tumors were often located in stomach $(n=9)$, followed by duodenum $(n=2)$, small intestine $(\mathrm{n}=2)$, pelvic cavity $(\mathrm{n}=1)$, esophagus 


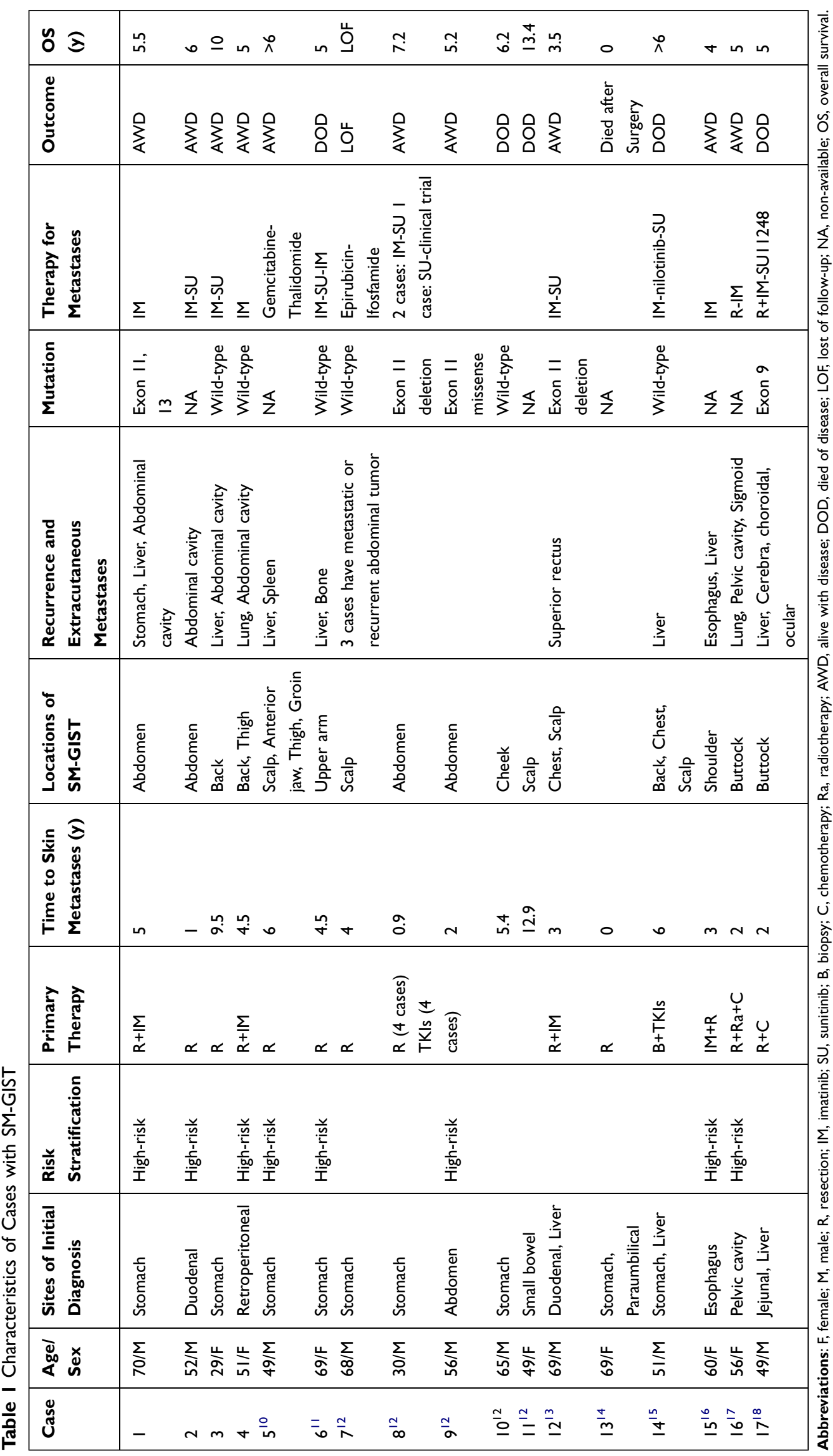


$(\mathrm{n}=1)$, abdomen $(\mathrm{n}=1)$ and retroperitoneal $(\mathrm{n}=1)$. A total of 4 cases had metastases at the initial diagnosis and all the 8 cases with available detailed information of the localized primary tumors can be classified into high-risk group according to the 2008 modified NIH consensus. SM-GIST mainly occurred in the head and face $(n=6)$, and extremities $(n=6)$, followed by abdomen wall $(n=5)$, back $(n=3)$ and chest $(n=2)$. Noteworthy, almost all the cases had metastases in other parts before or during the occurrence of SM-GIST. Eleven cases provided molecular analysis data, and wild-type $(n=6)$ was the most common subtype, followed by mutation in exon 11 $(n=4)$, exon $9(n=1)$ and exon $13(n=1)$.

Surgical resection was the main initial therapy $(n=16)$, and after surgery, 6 cases received TKIs and 2 cases received chemotherapy. The average time to SM-GIST was 4.22 years, specifically 4.59 years in gastric and 3.8 years in non-gastric cancer. Moreover, for resection only group (including chemotherapy) such average time was 3.63 years, while for the combined group (resection and TKIs) it was about 4.74 years.

For SM-GIST, treatment predominantly included TKIs, combined with or without operation, and 2 cases received chemotherapy. A total of 15 cases had available follow-up ( 1 case was lost to follow-up and 1 case died after surgery). The average survival of the 15 cases was approximately 6.2 years, while after the diagnosis of SM-GIST, the average survival was more than 1.69 years. The prognosis of the cases with exon 11 mutations was better than those with WT-GIST (average survival, 2.63 vs 0.46 years, respectively).

\section{Discussion}

\section{Skin Metastasis of GIST (SM-GIST)}

GISTs metastasize mainly to liver and abdominal cavity, while metastases outside the abdomen occurs only in advanced patients. $^{2}$ SM-GIST, in particular, occurs in approximately $1 \%$ of advanced patients, ${ }^{9}$ but a lower incidence has been reported in some studies. ${ }^{19,20}$ Maybe the reason is that, like our cases, skin nodules are the most frequent manifestations, and these nodules can be easily ignored due to their small size and asymptomatic patients. In this series, head and face and extremities were the most common sites for SM-GIST. This is largely because these areas are rich in blood supply and hematogenous metastasis is the preferred metastatic pathway of GIST, while the lymphatic pathway is uncommon. ${ }^{2}$
GIST has different malignant potentials which can be evaluated by the location, size and mitotic index (MI) of the primary tumors. ${ }^{21}$ Non-gastric GISTs and tumor with larger tumor size or higher MI have a higher risk of recurrence and metastasis. ${ }^{21}$ Like in our study, high-risk GIST is more likely to metastasize to the skin and gastric GIST has longer time to SM-GIST than non-gastric tumors. Tumor rupture is another important factor affecting prognosis. The 5-year recurrence-free survival (RFS) of patients with tumor rupture was $37 \%$, but that of patients with minor defects of tumor integrity and no defect was $91 \%$ and $97 \%$ respectively. ${ }^{22}$ Burkill et al reported 2 patients with subcutaneous metastases in relation to operation scars. ${ }^{23}$ Thus, we should pay attention to distinguish iatrogenic diffusion especially the SM-GIST near the surgical scar, as these two metastatic patterns have different clinical and indicative significance.

The molecular subtype of GIST also has different prognostic and predictive relevance. GISTs with exon 9 mutation seem to be more aggressive than those with exon 11 mutation, ${ }^{24,25}$ and this is also manifested in KIT exon 13 mutant. ${ }^{26}$ However, PDGFRA mutants and WT-GIST have lower metastatic potential than KIT mutants but they are less responsive to imatinib-based therapies and have a worse prognosis. ${ }^{24,27}$ Imatinib-resistance including primary and secondary resistance, is also a promoting factor. Tumor with KIT exon 9 and WT-GIST are resistant to IM at the beginning of the treatment. Although exon 11 mutation is sensitive to IM, secondary drug-resistance mutations will occur in the later stage. ${ }^{28}$ As shown in our study, KIT exon 9, 11 and WT-GIST seem to be conducive to skin metastasis.

The median postoperative time for distant metastases of GIST is about 2 years. ${ }^{29}$ However, in this study, the mean time to SM-GIST was 4.22 years. This discrepancy is related to that unlike carcinomas, which sometimes initially metastasize to the skin, SM-GIST is the late manifestation of disseminated diseases. A majority of patients had developed liver, abdominal metastasis before the occurrence of SM-GIST. Although the average survival was approximately 6.2 years, that was 1.69 years after diagnosis of SM-GIST. Similarly, Vandergriff et al reported that the mean survival in patients with cutaneous metastasis of leiomyosarcoma was only 10 months after diagnosis. ${ }^{30}$ Therefore, SM-GIST indicates a widespread systemic disease that already exist and a poorer prognosis. 


\section{Diagnosis of SM-GIST}

The differential diagnosis of SM-GIST is a diagnostic challenge in which the morphological differential diagnosis is broad and includes primary and metastatic tumors, such as sarcomatoid carcinoma and melanoma. ${ }^{12}$ About $10 \%$ of metastatic tumors can metastasize to the skin, among which breast cancer and melanoma are the most common. ${ }^{31}$ Currently, IHC is a significant supplementary tool for histopathology to confirm the diagnosis of GIST. GIST has unique IHC features and staining for CD117 and DOG1 can establish the diagnosis of GIST. ${ }^{45}$ In a study involving 1168 GIST patients, CD117 and DOG1 had identical diagnostic sensitivity $(94.7 \%$ and $94.4 \%$, respectively) and their positive coincidence was very high (92.3\% for both). ${ }^{32}$ Furthermore, DOG1 is also specific and sensitive for CD117-negative GISTs. ${ }^{7}$ Other IHC biomarkers including CD34 (70\%), SMA (30-40\%), S-100 protein $(5 \%)$ and desmin (1-2\%) also can be helpful for diagnosis. ${ }^{33}$ Markers of skin spindle cell tumors have been described in previous reports. ${ }^{12,15}$ If necessary, mutation analysis can also help confirm the diagnosis, which is also of paramount importance for the selection of the optimal therapy. ${ }^{34}$

\section{Dedifferentiation of GIST}

Dedifferentiation is a process involving morphological and immunophenotypic alterations associated with tumor progression. ${ }^{7}$ Pauwels et al first applied the term "dedifferentiation" to GISTs, and defined it as an alteration in the histological characteristics of the primary tumor, with the immunoreactivity of CD117 changing from positive to negative. ${ }^{35}$ Subsequently, CD34 and DOG1 were also shown not to be expressed in anaplastic components of dedifferentiated GIST. ${ }^{35,36}$ Several studies reported that dedifferentiation of GIST occurred only in patients with long-term imatinib treatment. ${ }^{35,37,38}$ This phenomenon can also occur in imatinib-naïve patients. ${ }^{39}$

Antonescu et al studied the potential molecular mechanism underlying GIST dedifferentiation. ${ }^{39}$ Their study included 3 imatinib-resistant and 5 imatinib-naïve patients who experienced transition from GIST with CD117-positive and spindle cell morphology to those with CD117-negative and anaplastic components. Molecular analysis showed that there was no difference in KIT mutations between the classical and anaplastic components, and 4 patients harbored mutation in KIT exon 11 and the other were wild-type for KIT, PDGFRA and $B R A F$ between two components analyzed. Compared to the conventional area, the anaplastic area harbored loss of one KIT in 3 patients and had low-level amplification of KIT in 2 patients. Therefore, the authors suggested that dedifferentiation of GIST may be caused by genetic instability involving low level of KIT amplification and loss of heterozygosity. ${ }^{39}$ One of our cases (case 4) had loss of expression of DOG1 in SM-GIST (Figure 2) but no anaplastic components, and surgery is not an optimal choice due to advanced disease. Although dedifferentiation cannot be confirmed and stained for DOG1 and CD117, the SM-GIST was also diagnosed by medical history and efficacy of TKIs. Furthermore, there are few reports on the treatment of GIST after dedifferentiation and our study provides further insights in this regard.

\section{Treatment}

Surgical resection is the gold standard for the treatment of localized GIST, which can cure approximately $60 \%$ of patients. ${ }^{40}$ The prognosis of GIST which is low- or medium-risk is relatively good, but the recurrence of high-risk tumors is almost inevitable after resection. ${ }^{29}$ Adjuvant imatinib, which has gradually become a supplement to surgical treatment, can reduce the risk of recurrence and metastasis, especially for the high-risk tumors. ${ }^{1}$ For patients with localized GIST, 3 year of adjuvant imatinib can improve RFS (5-year RFS, 65.5\%) and overall survival (OS) (5-year OS, 92.0\%), and similar imatinib regimen is also recommended for high-risk patients in the NCCN guide. $^{24,41}$ As shown in this study, resection combined with TKIs can delay the occurrence of SM-GIST.

Regarding the therapy for SM-GIST, what deserves our attention is that the treatment should focus on extracutaneous metastases, such as liver, abdominal cavity or lung instead of a skin lesion as it will not have a serious adverse effect on the function of the body. In addition, the resection of SM-GIST can play both diagnostic and therapeutic roles. Two Phase III studies confirmed the long-term efficacy of imatinib in patients with advanced GIST, with a median OS of more than 4 years. ${ }^{9,42}$ Furthermore, patients with KIT exon 11 mutation can benefit more from imatinib treatment than those with wild-type, PDGFRA and other KIT mutations. ${ }^{43}$ When resistance to imatinib, sunitinib and regorafenib are recommended as the second- and third-line drugs for advanced disease. ${ }^{24}$ Metastasectomy is controversial and should be considered carefully. Among the patients with responsive disease, the median progression-free survival (PFS) after surgery was 31 months, better than patients 
with stable disease, unifocal progression and multifocal disease (19, 10 and 5 months, respectively). ${ }^{44}$

In conclusion, primary tumor location, therapy for primary disease, risk stratification, tumor burden and molecular subtype may affect the occurrence of SM-GIST. When in doubt, clinicians should perform a comprehensive skin examination. IHC and molecular analysis can establish the diagnosis of SM-GIST. The focus of the treatment with TKIs is for extracutaneous metastases, and surgery should be performed if necessary.

However, our study has some limitations. First, due to the rarity of this condition, the number of patients in the study is relatively small. Second, the clinicopathological data of some patients are incomplete, which may deviate the results of the study. Third, this study is a retrospective study with possible selection bias. Fourth, the cases included in this study had a long-time span, and the treatment methods were different in different periods and centers. On the other hand, this is the first systematic retrospective study on SM-GIST. A larger sample size study is needed and we hope that this study will improve the clinical understanding of GIST and its skin metastasis.

\section{Ethics Statement}

The study was approved by the Ethics Committee of Xiangya Hospital of Central South University, and informed consent was obtained from the patients. The patients or the legal representative provided a written informed consent to the details and images of the cases to be published. The study is in line with the Helsinki Declaration and does not violate medical ethics.

\section{Acknowledgment}

This study was supported by the Hunan Provincial Clinical Medical Technology Innovation Guidance Project, China (2018SK52604).

\section{Disclosure}

The authors report no conflicts of interest in this work.

\section{References}

1. von Mehren M, Joensuu H. Gastrointestinal stromal tumors. J Clin Oncol. 2018;36(2):136-143. doi:10.1200/JCO.2017.74.9705

2. Corless CL, Fletcher JA, Henrich MC. Biology of gastrointestinal stromal tumors. J Clin Oncol. 2004;22(18):3813-3825. doi:10.1200/ JCO.2004.05.140

3. Miettinen M, Lasota J. Gastrointestinal stromal tumors: review on morphology, molecular pathology, prognosis, and differential diagnosis. Arch Pathol Lab Med. 2006;130(10):1466-1478. doi:10.1043/1543-2165(2006)130[1466:GSTROM]2.0.CO;2
4. Medeiros F, Corless CL, Duensing A, et al. KIT-negative gastrointestinal stromal tumors - proof of concept and therapeutic implications. Am J Surg Pathol. 2004;28(7):889-894. doi:10.1097/ 00000478-200407000-00007

5. West RB, Corless CL, Chen X, et al. The novel marker, DOG1, is expressed ubiquitously in gastrointestinal stromal tumors irrespective of KIT or PDGFRA mutation status. Am J Pathol. 2004;165 (1):107-113. doi:10.1016/S0002-9440(10)63279-8

6. Corless CL, Barnett CM, Heinrich MC. Gastrointestinal stromal tumours: origin and molecular oncology. Nat Rev Cancer. 2011;11 (12):865-878. doi: $10.1038 / \operatorname{nrc} 3143$

7. Karakas C, Christensen P, Baek D, Jung MJ, Ro JY. Dedifferentiated gastrointestinal stromal tumor: recent advances. Ann Diagn Pathol. 2019;39:118-124. doi:10.1016/j.anndiagpath.2018.12.005

8. Emile JF, Brahimi S, Coindre JM, et al. Frequencies of KIT and PDGFRA mutations in the MolecGIST prospective population-based study differ from those of advanced GISTs. Med Oncol. 2012;29 (3):1765-1772. doi:10.1007/s12032-011-0074-y

9. Verweij J, Casali PG, Zalcberg J, et al. Progression-free survival in gastrointestinal stromal tumours with high-dose imatinib: randomised trial. Lancet. 2004;364(9440):1127-1134. doi:10.1016/S01406736(04)17098-0

10. Shabahang M, Livingstone AS. Cutaneous metastases from a gastrointestinal stromal tumor of the stomach: review of literature. Digest Surg. 2002;19(1):64-65. doi:10.1159/000052009

11. Kroep JR, Bovee JVMG, van der Molen AJ, Hogendoorn PCW, Gelderblom H. Extra-abdominal subcutaneous metastasis of a gastrointestinal stromal tumor: report of a case and a review of the literature. J Cutan Pathol. 2009;36(5):565-569. doi:10.1111/ j.1600-0560.2008.01067.x

12. Wang WL, Hornick JL, Mallipeddi R, et al. Cutaneous and subcutaneous metastases of gastrointestinal stromal tumors: a series of 5 cases with molecular analysis. Am J Dermatopathol. 2009;31 (3):297-300. doi:10.1097/DAD.0b013e31818acb1a

13. Kim YJ, Lee WJ, Won CH, Choi JH, Lee MW. Metastatic cutaneous duodenal gastrointestinal stromal tumor: a possible clue to multiple metastases. Ann Dermatol. 2018;30(3):345-347. doi:10.5021/ ad.2018.30.3.345

14. Anagnostoulis S, Mimidis K, Papadopoulos V, et al. Subcutaneous metastasis from a gastrointestinal stromal tumor of the stomach: a case report. $J$ BUON. 2007;12(4):549-552.

15. Aickara DJ, McBride J, Morrison B, et al. Multidrug resistant gastrointestinal stromal tumor with multiple metastases to the skin and subcutaneous soft tissue: a case report and review of literature. J Cutan Pathol. 2020;47(4):398-401. doi:10.1111/cup.13611

16. Jones AP, Allison K. Subcutaneous metastasis of a GIST tumour unresponsive to imatinib. J Plast Reconstr Aesthet Surg. 2011;64 (9):e250-e251. doi:10.1016/j.bjps.2011.03.038

17. Pasku D, Karantanas A, Giannikaki E, et al. Bilateral gluteal metastases from a misdiagnosed intrapelvic gastrointestinal stromal tumor. World J Surg Oncol. 2008;6(1):139. doi:10.1186/1477-7819-6-139

18. Hughes B, Yip D, Goldstein D, et al. Cerebral relapse of metastatic gastrointestinal stromal tumor during treatment with imatinib mesylate: case report. BMC Cancer. 2004;4(1):74. doi:10.1186/14712407-4-74

19. Miettinen M, Makhlouf H, Sobin LH, Lasota J. Gastrointestinal stromal tumors of the jejunum and ileum - a clinicopathologic, immunohistochemical, and molecular genetic study of 906 cases before imatinib with long-term follow-up. Am J Surg Pathol. 2006;30(4):477-489. doi:10.1097/00000478-200604000-00008

20. Miettinen M, Sobin LH, Lasota J. Gastrointestinal stromal tumors of the stomach - a clinicopathologic, immunohistochemical, and molecular genetic study of 1765 cases with long-term follow-up. Am $J \quad$ Surg Pathol. 2005;29(1):52-68. doi:10.1097/01. pas.0000146010.92933.de 
21. Joensuu H. Risk stratification of patients diagnosed with gastrointestinal stromal tumor. Hum Pathol. 2008;39(10):1411-1419. doi:10.1016/j.humpath.2008.06.025

22. Hølmebakk T, Hompland I, Bjerkehagen B, et al. Recurrence-free survival after resection of gastric gastrointestinal stromal tumors classified according to a strict definition of tumor rupture: a population-based study. Ann Surg Oncol. 2018;25(5):1133-1139. doi:10.1245/s10434-018-6353-5

23. Burkill GJC, Badran M, Al-Muderis O, et al. Malignant gastrointestinal stromal tumor: distribution, imaging features, and pattern of metastatic spread. Radiology. 2003;226(2):527-532. doi:10.1148/ radiol.2262011880

24. Demetri GD, von Mehren M, Antonescu CR, et al. NCCN task force report: update on the management of patients with gastrointestinal stromal tumors. J Natl Compr Canc Ne. 2010;8:S1-S43. doi:10.6004/ jncen.2010.0116

25. Antonescu CR, Sommer G, Sarran L, et al. Association of KIT exon 9 mutations with nongastric primary site and aggressive behavior: KIT mutation analysis and clinical correlates of 120 gastrointestinal stromal tumors. Clin Cancer Res. 2003;9(9):3329-3337.

26. Lasota J, Corless CL, Heinrich MC, et al. Clinicopathologic profile of gastrointestinal stromal tumors (GISTs) with primary KIT exon 13 or exon 17 mutations: a multicenter study on 54 cases. Mod Pathol. 2008;21(4):476-484. doi:10.1038/modpathol.2008.2

27. Lasota J, Dansonka-Mieszkowska A, Sobin LH, et al. A great majority of GISTs with PDGFRA mutations represent gastric tumors of low or no malignant potential. Lab Invest. 2004;84(7):874-883. doi:10.1038/labinvest. 3700122

28. Liu P, Tan F, Liu H, et al. The use of molecular subtypes for precision therapy of recurrent and metastatic gastrointestinal stromal tumor. Onco Targets Ther. 2020;13:2433-2447. doi:10.2147/OTT.S241331

29. Rammohan A, Sathyanesan J, Rajendran K, et al. A gist of gastrointestinal stromal tumors: a review. World $J$ Gastrointest Oncol. 2013;5(6):102-112. doi:10.4251/wjgo.v5.i6.102

30. Vandergriff T, Krathen RA, Orengo I. Cutaneous metastasis of leiomyosarcoma. Dermatol Surg. 2007;33(5):634-637. doi:10.1111/ j.1524-4725.2007.33127.x

31. Lookingbill DP, Spangler N, Helm KF. Cutaneous metastases in patients with metastatic carcinoma: a retrospective study of 4020 patients. J Am Acad Dermatol. 1993;29(2 Pt 1):228-236. doi:10.1016/0190-9622(93)70173-Q

32. Miettinen M, Wang ZF, Lasota J. DOG1 antibody in the differential diagnosis of gastrointestinal stromal tumors a study of 1840 cases. Am J Surg Pathol. 2009;33(9):1401-1408. doi:10.1097/ PAS.0b013e3181a90e1a

33. Fletcher CDM, Berman JJ, Corless C, et al. Diagnosis of gastrointestinal stromal tumors: a consensus approach. Int J Surg Pathol. 2002;10(2):81-89. doi:10.1177/106689690201000201
34. Koo DH, Ryu MH, Kim KM, et al. Asian consensus guidelines for the diagnosis and management of gastrointestinal stromal tumor. Cancer Res Treat. 2016;48(4):1155-1166. doi:10.4143/crt.2016.187

35. Pauwels P, Debiec-Rychter M, Stul M, De Wever I, Van Oosterom AT, Sciot R. Changing phenotype of gastrointestinal stromal tumours under imatinib mesylate treatment: a potential diagnostic pitfall. Histopathology. 2005;47(1):41-47. doi:10.1111/j.13652559.2005.02179.x

36. Choi JJ, Sinada-Bottros L, Maker AV, Weisenberg E. Dedifferentiated gastrointestinal stromal tumor arising de novo from the small intestine. Pathol Res Pract. 2014;210(4):264-266. doi:10.1016/j. prp.2013.12.008

37. Díaz Delgado M, Hernández Amate A, Pereira Gallardo S, et al. Gastrointestinal stromal tumors: morphological, immunohistochemical and molecular changes associated with kinase inhibitor therapy. Pathol Oncol Res. 2011;17(3):455-461. doi:10.1007/s12253-0119362-2

38. Liegl B, Hornick JL, Antonescu CR, Corless CL, Fletcher CDM. Rhabdomyosarcomatous differentiation in gastrointestinal stromal tumors after tyrosine kinase inhibitor therapy a novel form of tumor progression. Am J Surg Pathol. 2009;33(2):218-226. doi:10.1097/ PAS.0b013e $31817 \mathrm{ec} 2 \mathrm{e} 6$

39. Antonescu CR, Romeo S, Zhang L, et al. Dedifferentiation in gastrointestinal stromal tumor to an anaplastic KIT-negative phenotype: a diagnostic pitfall: morphologic and molecular characterization of 8 cases occurring either de novo or after imatinib therapy. Am J Surg Pathol. 2013;37(3):385-392. doi:10.1097/PAS.0b013e31826c1761

40. Joensuu H, Vehtari A, Riihimäki J, et al. Risk of recurrence of gastrointestinal stromal tumour after surgery: an analysis of pooled population-based cohorts. Lancet Oncol. 2012;13(3):265-274. doi:10.1016/S1470-2045(11)70299-6

41. Joensuu H, Eriksson M, Hall KS, et al. One vs three years of adjuvant imatinib for operable gastrointestinal stromal tumor a randomized trial. JAMA-J Am Med Assoc. 2012;307(12):1265-1272. doi:10.1001/jama.2012.347

42. Blanke CD, Rankin C, Demetri GD, et al. Phase III randomized, intergroup trial assessing imatinib mesylate at two dose levels in patients with unresectable or metastatic gastrointestinal stromal tumors expressing the kit receptor tyrosine kinase: S0033. J Clin Oncol. 2008;26(4):626-632. doi:10.1200/JCO.2007.13.4452

43. Li GZ, Raut CP. Targeted therapy and personalized medicine in gastrointestinal stromal tumors: drug resistance, mechanisms, and treatment strategies. Onco Targets Ther. 2019;12:5123-5133. doi:10.2147/OTT.S180763

44. Fairweather M, Balachandran VP, Li GZ, et al. Cytoreductive surgery for metastatic gastrointestinal stromal tumors treated with tyrosine kinase inhibitors: a 2-institutional analysis. Ann Surg. 2018;268 (2):296-302. doi:10.1097/SLA.000000000002281

\section{Publish your work in this journal}

Cancer Management and Research is an international, peer-reviewed open access journal focusing on cancer research and the optimal use of preventative and integrated treatment interventions to achieve improved outcomes, enhanced survival and quality of life for the cancer patient.
The manuscript management system is completely online and includes a very quick and fair peer-review system, which is all easy to use. Visit http://www.dovepress.com/testimonials.php to read real quotes from published authors. 